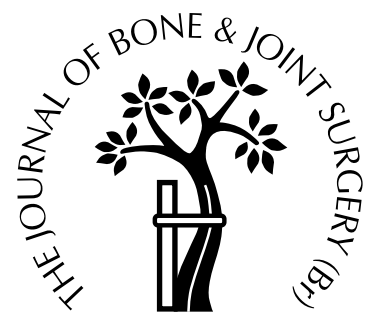

\title{
The use of dynamic interventional MRI in developmental dysplasia of the hip
}

\author{
S. Tennant, C. Kinmont, G. Lamb, W. Gedroyc, D. M. Hunt \\ From St Mary's Hospital, London, England
}

C Yonventional methods of imaging in the investigation of developmental dysplasia of the hip all have disadvantages, either in definition or in exposure to radiation. We describe a new open-configuration MR scanner which is unique in that it allows anaesthesia and access to the patient within the imaging volume for surgical procedures and application of casts. We performed 13 scans in eight anaesthetised infants. Dynamic imaging revealed two dislocated hips which were then visualised during reduction. Hip spicas were applied without removing the patient from the scanner. In one hip, an adductor tenotomy was carried out. In all patients, stressing the hips during dynamic imaging allowed an assessment of stability. This was particularly useful in two hips in which an analysis of stability in different positions facilitated the planning of femoral osteotomies. This method of imaging provides new and important information. It has great potential in the investigation of developmental dysplasia of the hip and, with ultrasound, may allow management without the need for radiography.

J Bone Joint Surg [Br] 1999;81-B:392-7.

Received 23 March 1998; Accepted after revision 29 September 1998

Safe, detailed and reproducible imaging in the management of developmental dysplasia of the hip remains a problem. Plain radiography, ultrasonography, arthrography, CT and MRI all provide valuable information, but each has its drawbacks. Plain-film radiography is limited by the inability to show soft tissues and repeated exposure to ionising radiation. ${ }^{1-9}$ Arthrography allows dynamic imaging to be achieved and is the yardstick against which other tech-

S. Tennant, FRCS, Orthopaedic Research Fellow

C. Kinmont, FRCS, Orthopaedic Registrar

G. Lamb, FRCR, Radiology Research Fellow

W. Gedroyc, FRCR, Consultant Radiologist

D. M. Hunt, FRCS, Consultant Orthopaedic Surgeon

St Mary's Hospital, Praed Street, London W2 1NY, UK.

Correspondence should be sent to Mr D. M. Hunt.

(C)1999 British Editorial Society of Bone and Joint Surgery 0301-620X/99/38964\$2.00 niques are measured. It is, however, an invasive procedure which involves considerable doses of ionising radiation and does not directly visualise the anatomy of the soft tissues in more than one plane. ${ }^{3,7,8}$ Ultrasonography is safe and multiplanar real-time imaging is possible. It can be used to monitor treatment in a harness, splint or hip spica, but interpretation is difficult and requires experience. ${ }^{\text {1,5,7-9 }} \mathrm{CT}$ and MRI are non-invasive and give excellent pictures, but $\mathrm{CT}$ requires substantial exposure to ionising radiation, ${ }^{10}$ and imaging is only possible in one plane. Conventional MRI has many advantages, as has been recently illustrated. ${ }^{11}$ It provides excellent soft-tissue discrimination and multiplanar imaging. Nevertheless, the technique is slow and only static imaging is possible. In young children general anaesthesia is required.

The open configuration MRI system which we describe combines the advantages of conventional MRI with access to the patient by the operator to move the limb. Dynamic imaging in near real-time can be achieved, allowing the analysis of bony and soft-tissue components while assessing stability. Immobilisation in a cast and some surgical procedures can be carried out within the imaging volume. These are all potential advantages over current methods of imaging and merit proper assessment. It is expensive, slow and, as yet, the true value of the images has not been determined. We describe a preliminary assessment of this technique in eight patients.

\section{Patients and Methods}

Eight patients (seven girls and one boy) received a total of 13 scans over a period of ten months. Their mean age was 16 months ( 3 to 38 ) at the time of the first scan.

We used an open configuration Signa SPIO MRI system (General Electric, Milwaukee, Wisconsin). In this system the coils of a 0.5 Tesla superconducting magnet are housed in separate, but communicating cryostats (Fig. 1). This provides a gap $56 \mathrm{~cm}$ wide in which the surgeon has access to the patient within the most homogeneous part of the static magnetic field. There are no radiofrequency coils in the gap. Instead, flexible surface coils act as both transmitters and receivers and can be wrapped unobtrusively around the part of the anatomy to be imaged. Imaging is achieved either in a conventional static mode, or in a real- 


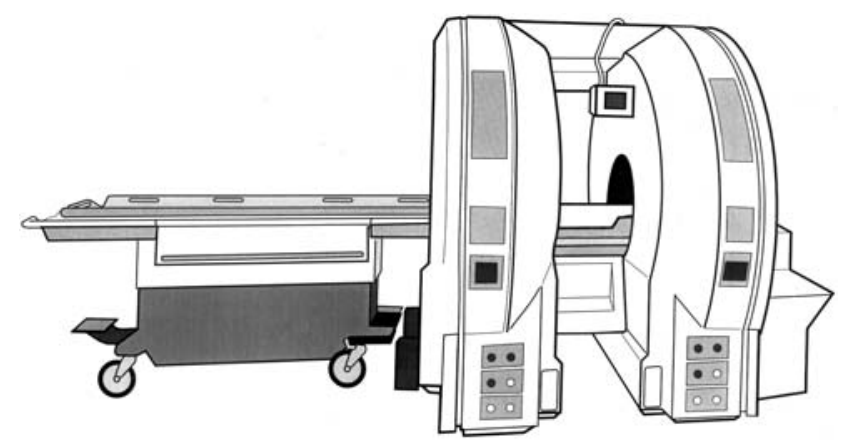

Fig. 1

Diagram of the open configuration of the MR imaging system Signa SPIO.

time mode with control of the image plane and position. $^{12-17}$ Rapid gradient-echo scans at a rate of one scan every two to four seconds are used.

General anaesthesia is required and longer ventilation tubing is needed to reach the infant. Induction of anaesthesia takes place within the scanner room on the sliding operating table outside the bore of the magnet. While the anaesthetic monitoring equipment (Maglife; Groupe Bruker Odam, Wissembough, France), ventilator (Penlon, Abingdon, UK) and Boyles machine (Ohmeda, Madison, Wisconsin) are specifically MR-compatible, many of the disposables are plastic and can be used as normally. The intubated infant is positioned on a specially made wooden hip spica table which rests on the main operating table (Fig. 2 ) and slides into the bore of the magnet. The caudal end of the special table tapers to a point and is raised by $10 \mathrm{~cm}$ from the base to support the sacrum. The legs can be left free or held in special holders to allow full assessment of hip movements, positioning of the surface coil and, if required, the application of a hip spica. A small, flexible single-loop surface coil is wrapped round the pelvis to optimise imaging of the hips. This coil is easily removed and replaced over a hip spica when required. Communica-

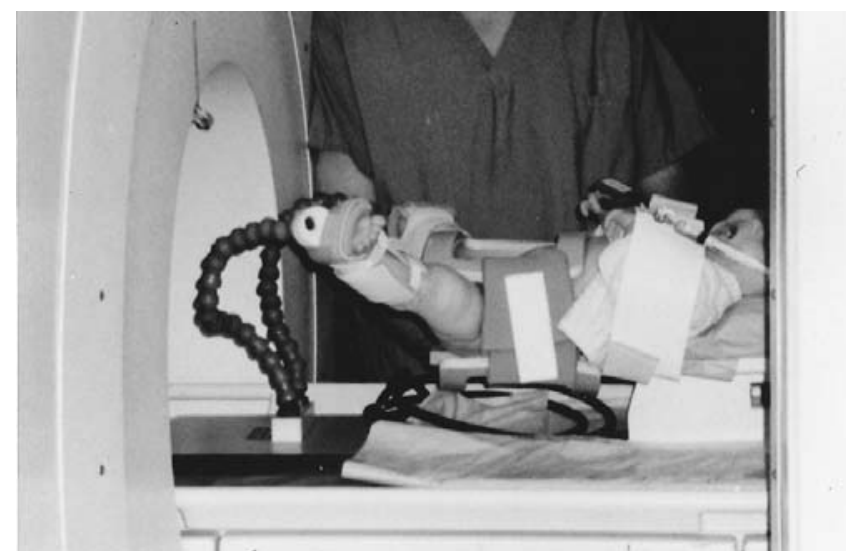

Fig. 2

Photograph of a child anaesthetised in the scanner on the wooden hip spica table. tion between the operator and the control room is maintained by a microphone and speaker. Two liquid-crystal MR-compatible monitors are mounted in the gap between the magnets and display the MR images to the operators.

With the scanner in static mode, coronal and axial T1weighted spin-echo images (TR 500, TE 19, FOV 24, all scans $4.0 \mathrm{~mm}$ thick, 256/192 matrix, 4 NEX) are initially obtained to assess the anatomy of the hip in detail. Dynamic imaging using a fast gradient-echo sequence is carried out for the evaluation of joint movement. Inversion-recovery prepared sequences are used which provide moderate T1-weighting. Rapid scans can then be obtained to assess the position of the hip as the femur is moved, with rapid switching between the coronal and axial planes, the axial images being the most useful. It was found that the best near real-time images were achieved by the operator stabilising the pelvis with one hand and moving the abnormal hip while a single image centred on the femoral head of the normal hip was repeated every two seconds. Further static spin-echo images of the hip may be obtained after a spica is applied. When imaging and application of a cast have been completed, the infant is withdrawn from the magnet on the imaging table.

\section{Results}

In two hips dynamic imaging revealed a dislocated but reducible hip which was visualised during reduction. Hip spicas were applied without removing the patient from the scanner. In one hip, an adductor tenotomy was carried out within the imaging volume. In all patients, stressing the hips during dynamic imaging allowed an assessment of stability to be made. This was particularly useful in two hips in which an analysis of stability in different positions facilitated the planning of subsequent femoral osteotomies.

One patient (case 2) demonstrated hip morphology which we believe has not been shown before. This infant was born with a dislocatable right hip which failed to relocate and stabilise after treatment in a Pavlik harness.

At the age of five months she underwent a period of Gallows traction. Examination under anaesthesia and screening under image intensifier showed a locatable right hip which was more stable after an adductor tenotomy. After three months in a hip spica, plain radiography revealed a small femoral head reduced in a dysplastic acetabulum. Dynamic MRI demonstrated a small, concentrically reduced right femoral head in a shallow acetabulum. The femoral head subluxated anteriorly on hip abduction. While evident on individual inversion-recovery prepared images, the anterior subluxation was best seen on the ciné loop of these images. Clinically, the femoral head was palpable anteriorly demonstrating a 'lump' sign. ${ }^{18}$ This can be seen on the MR scan (Fig. 3). A splint was applied under imaging control and maintained for three months. After its removal, plain radiography showed that the right hip had redislocated. She then had a second dynamic MR 


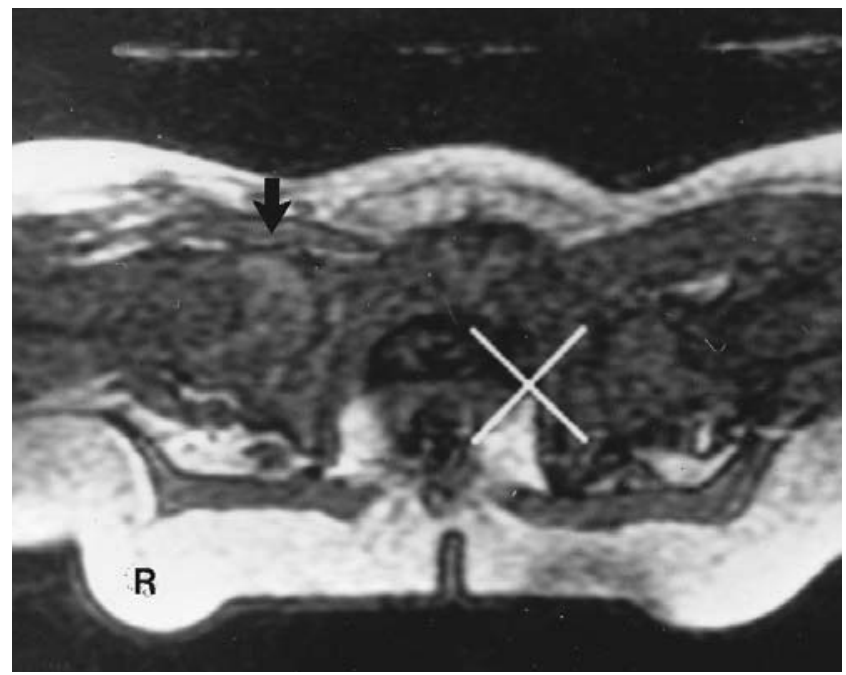

Case 2. Figure 3 - Axial MR scan demonstrating the 'lump sign' of the right hip (arrow). Figure 4 - Axial MR scans showing a) the right hip in flexion with posterior subluxation and $b$ ) internal rotation with the right femoral head located and well contained.

Case 4. Figure 5 - Axial MR scan showing a) external rotation of the right hip with a shallow acetabulum and anterior subluxation of the femoral head, and b) the position of the femoral head in varus and internal rotation.

Fig. 3

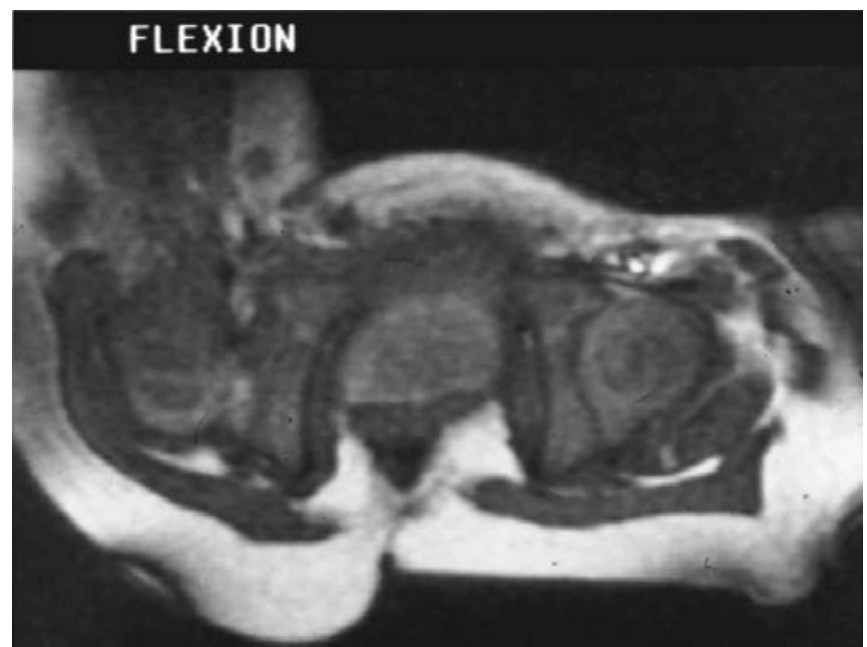

Fig. 4a

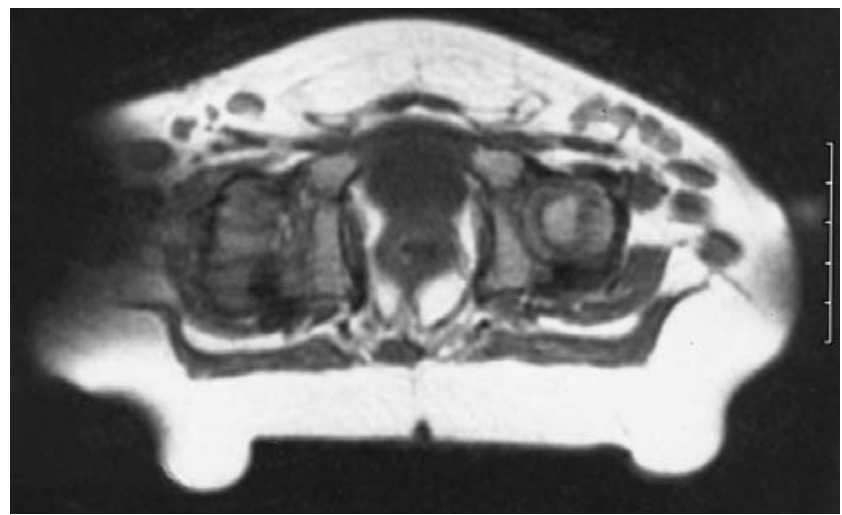

Fig. 5a

scan nine months after the first. This showed a good acetabulum with a hip which was located and stable in internal rotation but dislocated in flexion (Fig. 4). The dynamic scan was helpful in assessing the best position for stability. A varus derotation osteotomy has been carried out and the hip remains stable.

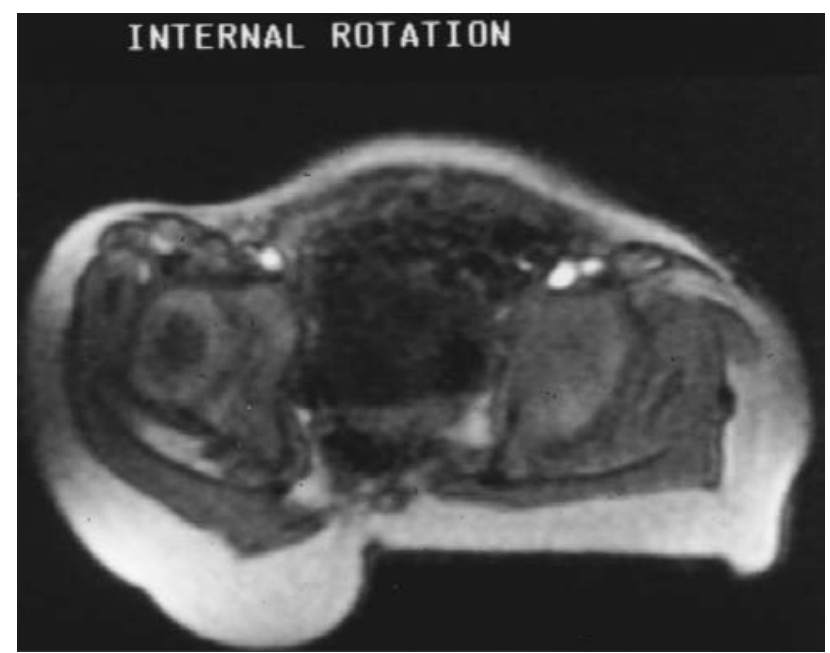

Fig. 4b

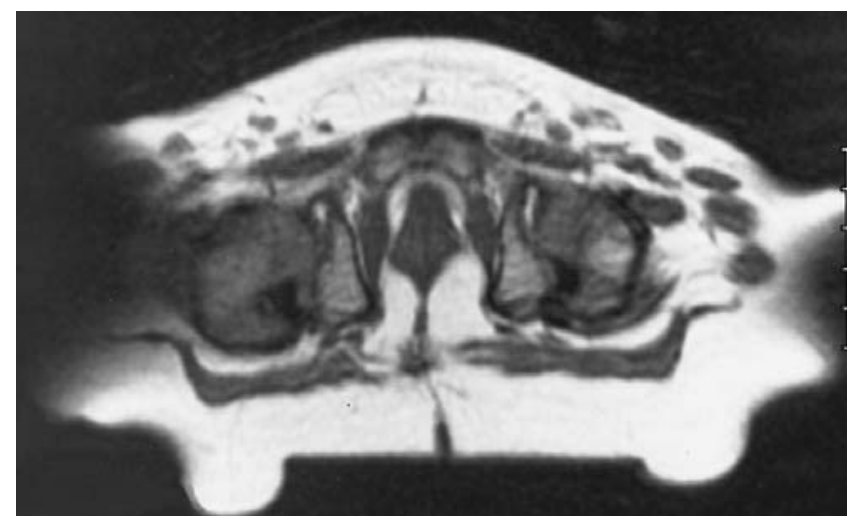

Fig. $5 b$

In another patient (case 4), a boy with a chromosomal disorder, dynamic MRI helped to plan an appropriate femoral osteotomy. He had had a difficult, resistant, dislocating right hip. Conservative treatment failed, and at 13 months he had an open reduction and a Salter innominate osteotomy. When his hip spica was removed there was concern 


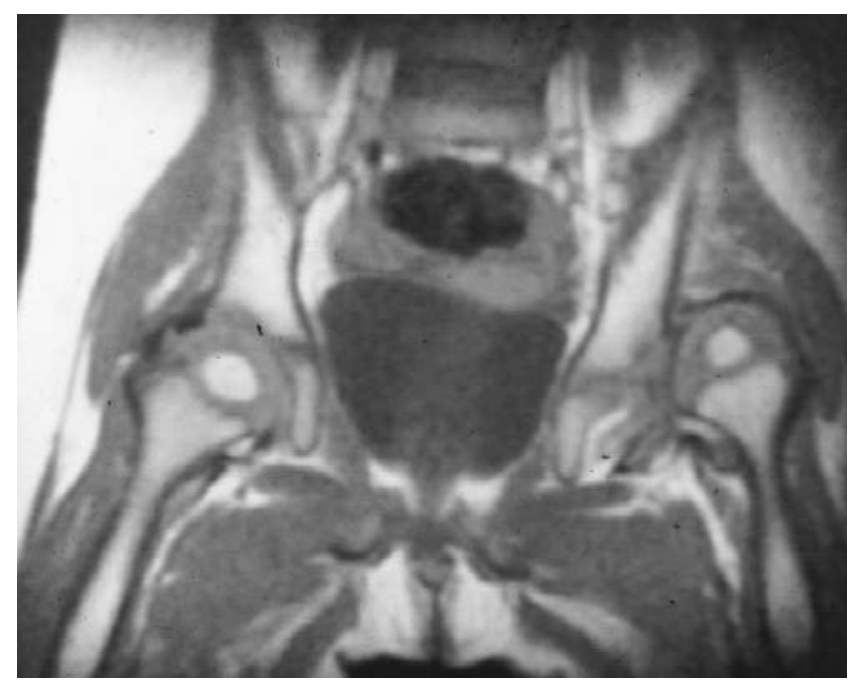

Fig. 6

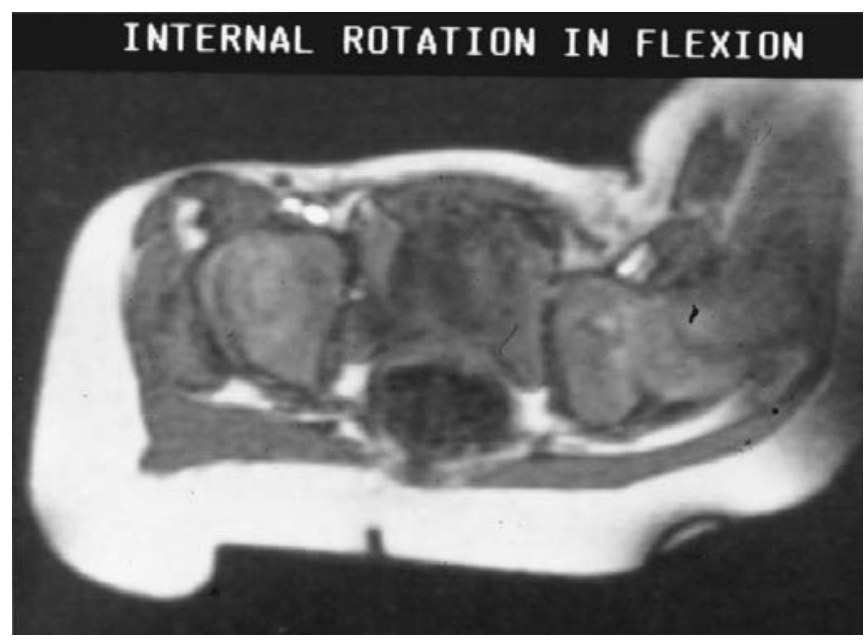

Fig. $7 \mathrm{a}$

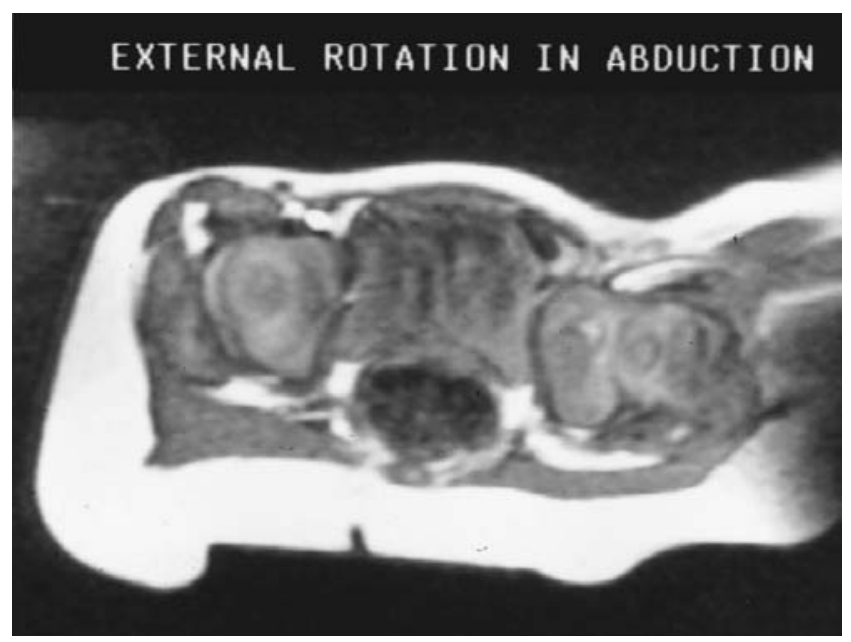

Fig. 7c that he still had an unstable hip and he therefore had dynamic MR imaging. T1-FSE-images showed that the femoral head was reduced in the realigned acetabulum, but was still unstable anteriorly. Inversion-recovery prepared real-time imaging (Fig. 5) allowed the optimum position for containment of the femoral head to be determined, so that an appropriate femoral osteotomy could be planned. This has been done and he is now walking with a stable, but still dysplastic hip.

In the final patient (case 8) dynamic MRI showed correct positioning of the femoral head in a very small, rudimentary acetabulum. Arthrography was also carried out and the information obtained from both techniques could be compared. The girl had been referred when 18 months old with an abnormal gait. She was found clinically to have a dislocated left hip and was treated in Gallows traction. She then had dynamic MR imaging. The acet-

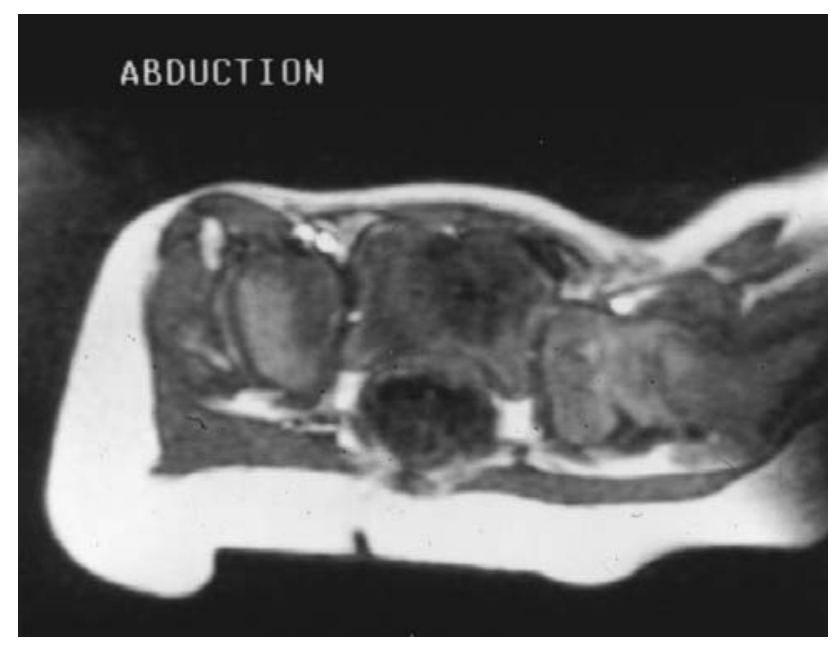

Fig. $7 b$

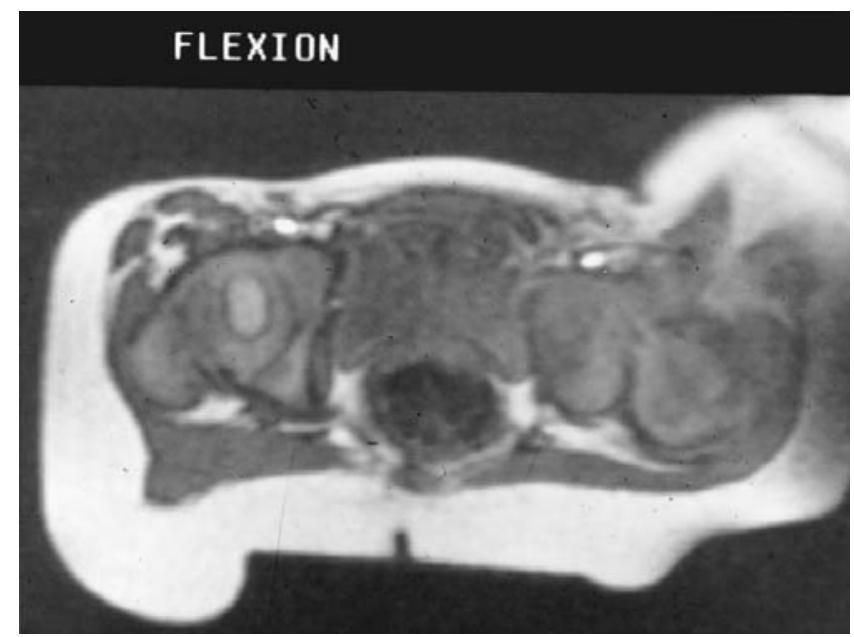

Fig. $7 \mathrm{~d}$

Case 8. Figure 6 - Coronal MR scan showing almost no acetabular cup on the left. The labrum is starting to form a false acetabulum. Figure 7 - MRI showing a) a coronal scan with the acetabulum on the left, conforming to the femoral head and an improved position in internal rotation with flexion, b) an axial scan with the position of the left hip improved further with abduction, c) an axial scan with the acetabular shape and position of the femoral head further improved on external rotation in abduction, and d) an axial scan with a very shallow acetabulum on the left and the femoral head lying dislocated posteriorly in flexion. 
abulum was virtually non-existent and a false acetabulum was seen to be developing superiorly (Fig. 6). The hip was found clinically to reduce into the rudimentary, but normally positioned acetabulum on internal rotation in flexion (Fig. 7a), which was confirmed on dynamic imaging. It was also stable in abduction in any position of rotation (Figs $7 \mathrm{~b}$ to d) but very unstable on flexion. A plaster spica was applied within the scanner, after which static images confirmed reduction. A subsequent arthrogram has confirmed the reduction but did not show the instability in flexion (Fig. 8).

\section{Discussion}

The new open configuration Signa SPIO is a 0.5 Tesla superconducting MR scanner and is the first of a new generation which offers considerable advantages over a conventional magnet, giving access to the patient in the scanner within the imaging volume. This allows simultaneous manipulation or intervention and scanning, with the facility for near real-time imaging to show movement of the joint and to examine it under stress for stability. The excellent definition of the cartilaginous acetabulum and femoral head allow a detailed assessment of their anatomy and relationship to one another. Both fast-spoiled gradientecho sequences and inversion-recovery prepared sequences used during the dynamic imaging provide mainly T1weighted images. The imaging can be repeated after application of a spica without moving the infant. In addition, the development of MR-compatible anaesthetic equipment has facilitated safe general anaesthesia of infants within the scanner.

The machine is large and has to be housed in an operating theatre. Communication between members of the surgical team is more difficult. The magnet limits direct lines of vision and creates considerable noise during imaging. A microphone-speaker system is required to link the surgeon with the control room. Close monitoring of the infant during anaesthesia is difficult since the head is concealed within the bore of the magnet and is only seen on a video camera. It is expensive, combining the costs of MRI with that of an operating-theatre. The presence of strongly ferromagnetic implants that may move or dislodge within the magnetic field is considered a contraindication to MRI. $^{8,19-21}$ A standard screening questionnaire is completed by the parent before surgery to minimise this risk, although metallic implants are rare in infants. This risk applies equally to surgical equipment which is brought in to the scanner room. All instruments are screened using a hand-held magnet before being allowed close to the main magnet. Particular care must be observed when using a scalpel with a ferromagnetic blade during an adductor tenotomy.

When undertaking invasive procedures within the scanner the problem of access caused by the surface coil and the potential image distortion by metallic instruments during scanning must be considered. We have tried to make an objective appraisal of the potential of interventional MR scanning in the management of developmental displacement of the hip. The indications are broadly the same as for arthrography and patients were selected on this basis so that a general anaesthetic was justified. In all these cases much of the information could have been obtained from ultrasound and arthrography. It was interesting to visualise reduction of the hip in four patients, although this was unexpected. There was concern about damage to the femoral head as seen in one patient on subsequent radiological examination.

Relocation can be seen on screening under image intensifier. The only real advantage was that this was visualised without radiography, and possibly provided better evidence for relocation in a very unstable hip. For those experienced in arthrography, MRI may not provide any more information. For the less experienced, the easier interpretation and the axial views make the pathology easier to understand. For example, it can be clearly demonstrated that the labrum obstructs relocation circumferentially, not just superiorly, as seen on arthrography (Fig. 9).

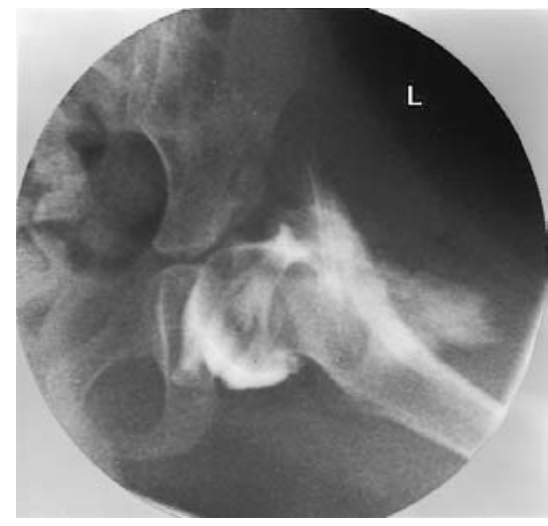

Fig. 8

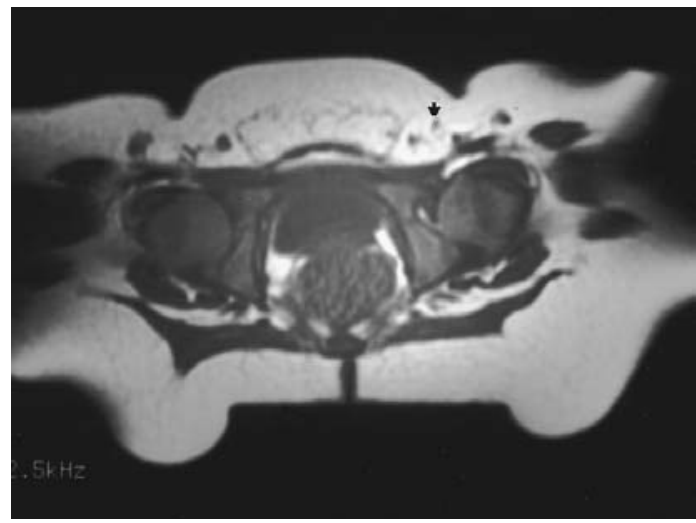

Fig. 9

Case 8 . Figure 8 - Arthrogram of the left hip showing the hip reduction but no instability in flexion. Figure $9-$ Axial view of the small acetabulum with head located but obstructed by the inturned labrum anteriorly. 
There is also the potential to carry out MR-guided surgical procedures. We have undertaken adductor tenotomies. This could be extended to psoas tenotomy and even a limited open reduction, but clearly this must be approached with caution. The most valuable information was that which helped to plan the femoral osteotomies in two patients. In the final patient (case 8) in which the axial views showed that the hip was unstable in flexion, it was clear that any form of acetabular reconstruction which compromised the posterior acetabular wall would be likely to fail.

Our study has shown that operator-controlled, near realtime dynamic interventional resonance MRI under general anaesthesia can produce good images of the pathological hip in very young infants. This is an early machine, which at the time of writing, is the only one in the UK. Others will follow; its use in other fields of medicine and surgery is well established. As the machines become more powerful and as near real-time becomes real-time, it has the potential to become a very useful tool in the imaging of developmental dysplasia of the hip.

No benefits in any form have been received or will be received from a commercial party related directly or indirectly to the subject of this article.

\section{References}

1. Boal DK, Schwenkter EP. The infant hip: assessment with real-time US. Radiology 1985;157:667-72.

2. Bos CFA, Bloem JL. Treatment of dislocation of the hip, detected in early childhood, based on magnetic resonance imaging. J Bone Joint Surg [Am] 1989;71-A:1523-9.

3. Bos CFA, Bloem JL, Oberman WR, Rozing PM. Magnetic resonance imaging in congenital dislocation of the hip. J Bone Joint Surg [Br] 1988;70-B:174-8.

4. Gabuzda GM, Renshaw TS. Reduction of congenital dislocation of the hip. J Bone Joint Surg [Am] 1992;74-A:624-31.

5. Graf R. Classification of hip joint dysplasia by means of sonography. Arch Orthop Trauma Surg 1984;102:248-55.
6. Guidera KJ, Einbecker ME, Berman CG, et al. Magnetic resonance imaging evaluation of congenital dislocation of the hips. Clin Orthop 1990;261:96-101.

7. Harcke HT. Imaging in congenital dislocation and dysplasia of the hip. Clin Orthop 1992;281:22-8.

8. Harcke HT, Kumar SJ. The role of ultrasound in the diagnosis and management of congenital dislocation and dysplasia of the hip. $J$ Bone Joint Surg [Am] 1991;73-A:622-8.

9. MacDonald J, Barrow S, Carty HM, Taylor JF. Imaging strategies in the first twelve months after reduction of developmental dislocation of the hip. J Paediatr Orthop B 1995;4:95-9.

10. Shrimpton PC, Jones DG, Hillier MC, et al. Survey of CT practice in the United Kingdom, part 2: Dosimetric aspects. National Radiological Protection Board 1991.

11. McNally EG, Tasker A, Benson MK. MRI after operative reduction for developmental dysplasia of the hip. J Bone Joint Surg [Br] 1997; 79-B:724-6.

12. Fried MP, Hsu L, Topulos GP, Jolesz FA. Image guided surgery in a new magnetic resonance suite: pre-clinical considerations. Laryngoscope 1996;106:411-7.

13. Jolesz FA, Silverman SG. Interventional magnetic resonance therapy Sem Int Radiol 1995;12:20-5.

14. Lufkin RB. Interventional MR imaging. Radiology 1995;197:16-18.

15. Moriarty TM, Kikinis R, Jolesz FA, Black PM, Alexander E. Magnetic resonance imaging therapy: intra-operative MR imaging. Neurosurg Clin N Am 1996; 7:323-31.

16. Schenck JF, Jolesz FA, Roemer PB, et al. Superconducting open configuration MR imaging system for image-guided therapy. Radiology 1995; 195:805-14.

17. Silverman SG, Collick BD, Figueira MR, et al. Inter-active MRguided biopsy in an open configuration MR imaging system. Radiology 1995;197:175-81.

18. Catterall A. Assessment of adolescent acetabular dysplasia. Recent advances in orthopaedics. no. 6, Churchill Livingstone, 1992: 103-17.

19. Kagetsu NJ, Litt AW. Important considerations in measurement of attractive force on metallic implants in MR imagers. Radiology 1991; 179:505-8.

20. New PF, Rosen BR, Brady TJ, et al. Potential hazards and artefacts of ferromagnetic and non-ferromagnetic surgical and dental materials and devices in nuclear magnetic resonance imaging. Radiology 1983; 147:139-48.

21. Shellock FG, Morisoli S, Kanal E. MR procedures and biomedical implants, materials and devices: 1993 update. Radiology 1993;189: 587-99. 